Received: November 1, 2017

Revision received: March 12, 2018

\title{
Application of Android System Software in English Listening and Speaking Teaching
}

\author{
Na Wang ${ }^{1}$ \\ Jiao Zuo Teachers College
}

\begin{abstract}
In view of the current imperfect functions of mobile English listening and speaking learning software and poor control performance, this paper designs and implements a set of English listening and speaking independent learning software based on Android system. In addition to the conventional audio file playback function, the software also implements audio file re-reading and subtitle matching, spoken language recognition and spoken language matching, lock screen control and other functions. The software effectively avoids the shortcomings of the current related software, expands the functions of the existing software. It has greatly improved the efficiency of English listening and speaking teaching.
\end{abstract}

\section{Keywords}

Listening and Speaking Teaching $\bullet$ Android System Software $\bullet$ Listening Training $\bullet$ Oral Training $\bullet$ Resource Library

${ }^{1}$ Correspondence to: Na Wang (MA), Jiao Zuo Teachers College, Jiaozuo 454000, China. Email: jzlwf@126.com 
The process of globalization has made us enter an era of learning English for all. Chinese people are faced with the dilemma of "dumb English" when they learn English (James, Matthew \& Samrachana, 2018; Wójcik, 2016; Liu \& Chu, 2010). They can't understand it at the same time. In the 1990s, users mainly used repeaters and tape recorders to practice listening. By the beginning of the 21 st century, users could practice listening and speaking on a personal computer.

In November 2007, the Open Handset Alliance, led by Google Inc., released the Android mobile operating system, which is an open source mobile operating system based on the Linux platform (Rockinson-Szapkiw \& Walker, 2009). Android system relies on its powerful features, easy operation, open source and other features to make more and more users use. According to Ai Media Consulting data, Android system reached $68.6 \%$ in China's smart phone market in 2012, which is It is possible for users to use Android phones for mobile English learning.

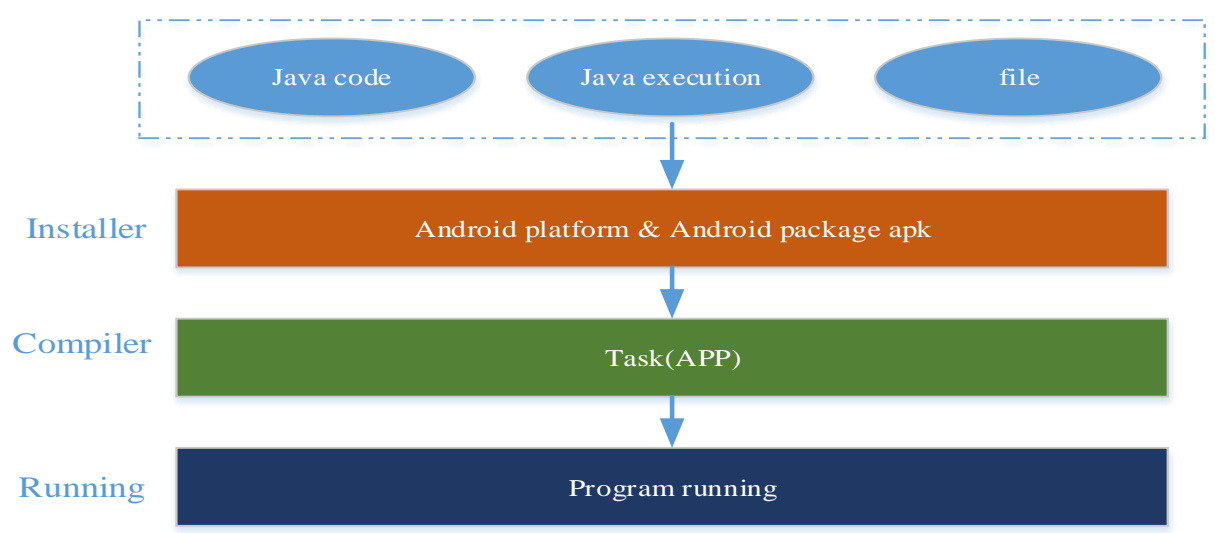

Figure 1. Android platform service.

As shown in figure 1, the upper layer of the seamless broadcaster is the broadcast control software, which converts the program broadcast commands into specific operations and sends them to the seamless broadcaster. The seamless broadcaster executes various commands of the broadcast control software and feedback the execution results (Filippo, 2016).

DirectShow architecture is a streaming media solution based on windows platform, which provides a mature solution for audio and video capture and playback. It can support many kinds of streaming media file formats, ASF files, MPEG files, AVI files, MP3 files and WAV files. At the same time, DirectShow architecture also supports the collection of multimedia data streams using WDM driver or earlier VFW driver. DirectShow architecture includes other published DirectX technologies, which can automatically detect and use audio and video hardware to accelerate streaming media playback, and it can also support systems without hardware acceleration. DirectShow technology greatly simplifies the playback of streaming media data, the format conversion of streaming media data and the collection of streaming media data (Bernstein, Eberhardt \& Jr, 2014; Rice, 2010). Not only that, the technology also provides a framework for developers to customize streaming media solutions in order to facilitate developers to create a DirectShow component that supports 
custom file formats or other uses. It has the following advantages: DirectShow technology can efficiently deal with the large amount of data streaming media information, and DirectShow technology can effectively maintain audio and video synchronization (Al-Otaibi, Alamer \& Al-Khalifa, 2016; Ou-Yang \& Wu, 2017; Cavus \& Ibrahim, 2017). In addition, DirectShow can handle a variety of media formats.

\section{Key technologies for mobile learning system development}

\section{J2ME technology}

The system was developed using J2ME (Java 2 Micro Edition), a technology introduced in Java for mobile communication devices, embedded devices or consumer appliances. Due to open technology and open source code, the system can be implemented across platforms (Wu, Lee, Chang \& Liang, 2013; Yen, Tsai \& Wu, 2013). Based on the Android platform, the mobile learning system is more suitable for J2ME technology development, and can be easily migrated to other platforms such as Windows Phone, and can also quickly pass local protocols. The information stored in the client is called out for the user to browse, and the information is downloaded from the Internet when the system needs it, saving traffic and reducing bandwidth occupation.

\section{OAUTH technology}

OAUTH is a secure, open and simple user resource license agreement. All major Internet companies provide OAUTH authentication services. The mobile learning system uses OAUTH authentication, which has the right to access user-authorized resources. Information will not be leaked and security will be high.

\section{Android background service technology}

Service is a service that runs in the background and does not interact with users. Service itself can not run, you need to call through Activity or other Context object, use the Context.start Service () method to start Service in the mobile learning system client. The student Android client running the background service can start up quickly and ensure that the push information is received in time.

\section{RSS and SAX technology}

RSS (Really Simple Syndication) is a data exchange specification for sharing news and other web content. It originated from Netscape Communications' push "Push" technology, which delivers customer-subscribed content to mobile learning system clients via RSS technology. Using SAX for parsing XML passed in RSS is a faster, lighter way to read and manipulate XML data. 


\section{Android mobile learning system overall architecture}

Figure 2 shows the overall architecture of the mobile learning system. The system uses a three-tier $\mathrm{C} / \mathrm{S}+\mathrm{B} / \mathrm{S}$ mode architecture. Students use the Android client to log in via WIFI/3G/4G wireless network and use OAUTH authentication for RSS subscriptions and course resources. The teacher uses the WWW browser in the PC to manage the mobile learning system.

The mobile learning system is developed in a three-tier structure. As shown in Table 1, the first layer is the user layer, which is divided into a PC client and an Android client, which is responsible for the design of the user interface; the second layer is the business logic layer, including the application server. For example, Tomcat is used to build the Web server layer; the third layer is responsible for data access for the data operation layer, the server database is built with MYSQL, and the Android mobile client uses lightweight sqlite3, which is consistent with the small amount of data stored by the client.

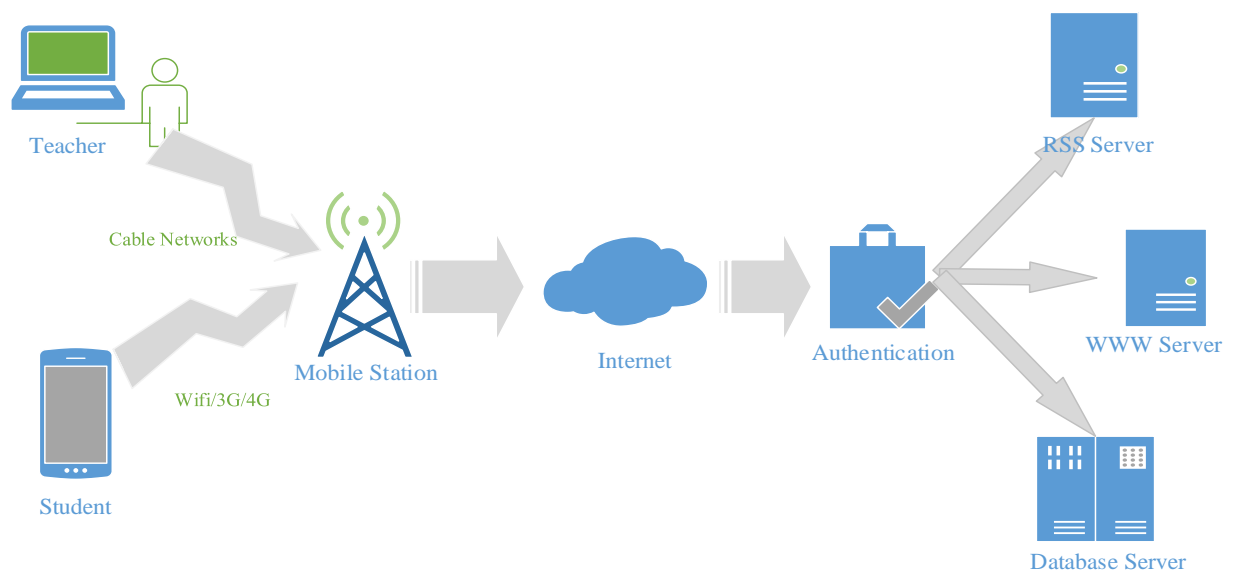

Figure 2. Mobile learning system architecture of Android mobile network.

Table 1

System Architecture Level

\begin{tabular}{ll}
\hline Logical layering & \multicolumn{1}{c}{ Physical layering } \\
\hline User layer & Android client, PC client \\
Business logic layer & application server \\
Data manipulation layer & Application library server MYSQL, client sqlt3 \\
\hline
\end{tabular}

\section{System requirements and design}

\section{System requirements analysis}

Functional requirements According to the shortcomings of the existing software mobile English learning software and the needs of users, the functional requirements are analysed from three aspects: listening, selflearning, listening training, speaking training and setting. 
1) Listening training. Listening training should have the usual functions of "play/pause", "fast forward/rewind", "previous song/next song", etc., and also need to include the listening material repeat function. A repeating function can be used to repeatedly play a certain segment of the listening material. During listening training, the Android system will enter the screen saver standby state after a period of time. In order to be able to control the English listening and speaking learning software when the mobile phone is unlocked, it is necessary to add a lock screen control function to the system, so that the user can also lock the screen state. Control the learning software. In order to facilitate users to classify listening audio files from different sources, the software needs to add functions such as adding grouping, editing grouping, deleting grouping and importing files to realize classified management of listening audio files.

2) Oral training. The user can record and save the English spoken by himself, and can play back and repeat the operation. At the same time, the software can automatically recognize the content according to the user's spoken language, judge the degree of matching between the spoken content and the listening material, and can feed the matching result to the user.

3) System settings. To enrich the user control experience, multiple system parameters need to be set to be dynamically adjustable. The parameters that the system can set include: Allow remote control settings, lock screen control settings, fast forward seconds settings, fast reverse seconds settings, playback mode settings, and my spoken storage directory settings.

User experience requirements A good Android app should have a good user experience in addition to its powerful features.

1) Friendly user interface. The user interface is directly contacted with the user as a system. The user interface is directly related to the user's use efficiency. A bad user interface can make the user feel disgusted with the system.

2) Quick response. The quick response means that the user must have a quick response when manipulating the system. The repeat function in the software requires a higher response time of the system, and the state transition of the program should be continuous and smooth.

3) Good handling. Nowadays, there are a wide variety of mobile applications, and more than three mobile applications can achieve the same function. In this case, only mobile applications are easier to use and easier to control.

\section{Module design}

According to the requirements analysis in Section 3.1, the English listening and speaking self-learning software is divided into the client and server 2, and the specific functional modules are shown in Figure 3.

1) Client. The English listening and speaking self-learning software client is based on the Android system development. The client is divided into four modules: listening training, oral training, resource downloading and system setting. Listening training is the core module of the system. The module is shown in Figure 2. The 
repeating function in listening training is realized by $\mathrm{AB}$ repeat. When the listening material is playing, click the $\mathrm{AB}$ repeat key to record the starting position of the listening material. At the end of the material segment, you only need to click the $\mathrm{AB}$ repeat button again to implement the loop repeat function. To switch to the normal play mode, click the $\mathrm{AB}$ repeat button.

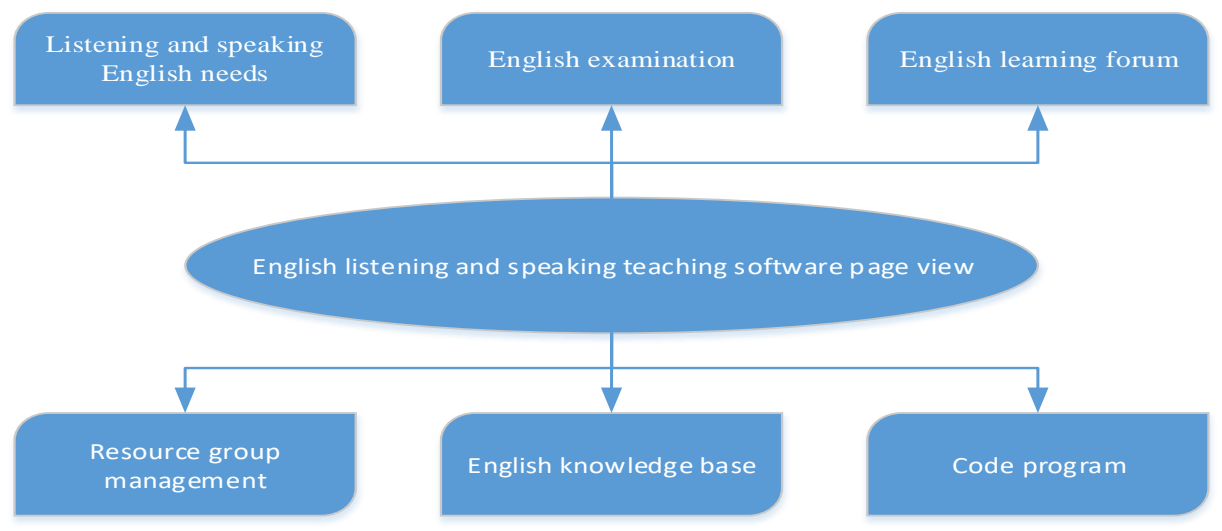

Figure 3. Overall design structure.

2) Server side. The English listening and speaking self-learning software server is mainly responsible for providing listening and speaking training materials for the Android client, so as to accurately and quickly respond to the client's request for listening and speaking materials. The server side is divided into a resource group management and a resource management module.

\section{Client interface design}

According to the design of the function module in Section 3.2, this section mainly designs the client interface. The system interface structure is shown in Figure 4.

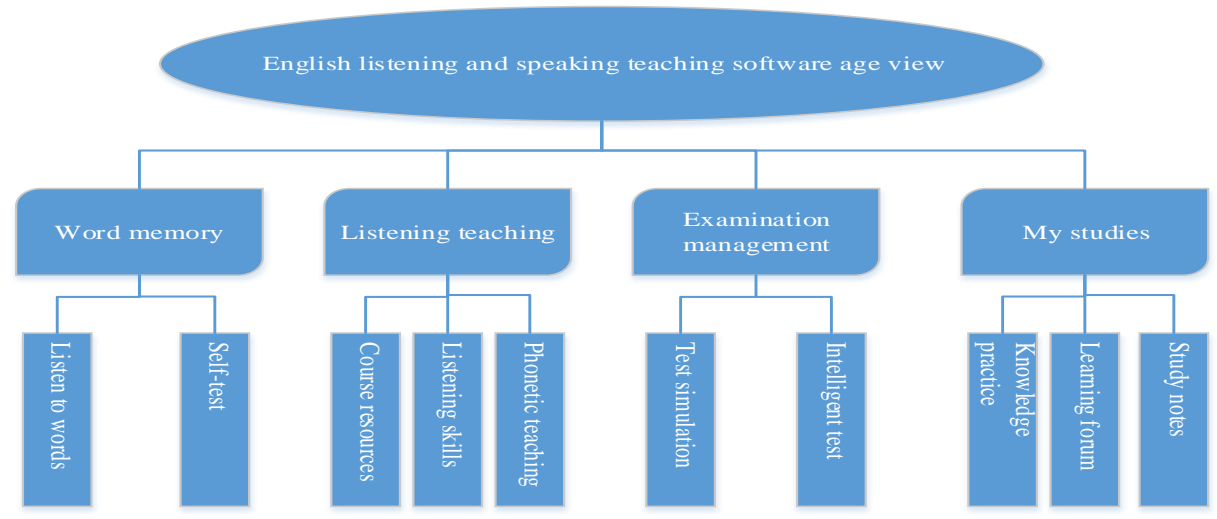

Figure 4. Teaching system function design. 
The entry system is divided into four main interfaces: Listening, Speaking, Resources and Settings. These are the listening interface, the oral training, the resource library and the setting interface of the function module. The four interfaces are switched by the Tab component in Android. All interfaces in the software automatically switch to the lock screen control interface when the Android system enters the screen saver program.

\section{System implementation}

\section{Listening training function}

The audio file playback function is implemented using the Media Player component of the Android system. Media Player is based on the Open Core underlying library, which enables Audio and Video playback. In the listening training function, the listening material re-reading function is one of the characteristics of the software. The user can repeatedly play a certain part of the listening material through the repeat function during the listening training, which provides an effective solution for this typical listening training method. The $\mathrm{AB}$ repeat is implemented using the Seek To method of the Media Player component, which specifies the start time of the audio file by setting the Seek-To parameter.

\section{Speaking training function realization}

The spoken language matching module adopts the form of a situational dialogue, and the resource library pre-sets different scene modes such as airport boarding and hotel occupancy. First, the user selects a spoken language scenario, and then the software automatically pronounces the voice according to the user selected scene, and then the user responds to the pronunciation of the system, and the system recognizes and matches the spoken language spoken by the user. The spoken language recognition function is implemented by calling the API interface of Google speech recognition. We can see the results in Table 2.

Table 2

Test result

\begin{tabular}{lcll}
\hline Module & $\begin{array}{c}\text { Test case } \\
\text { number }\end{array}$ & \multicolumn{1}{c}{ Operating } & \multicolumn{1}{c}{ Result } \\
\hline $\begin{array}{l}\text { Word memory } \\
\text { Listening } \\
\text { classroom }\end{array}$ & 1 & $\begin{array}{l}\text { Click the play button to listen to the phonetic } \\
\text { words } \\
\text { Click on the grammar to add the corresponding } \\
\text { text }\end{array}$ & $\begin{array}{l}\text { Achieve } \\
\text { expectations } \\
\text { Achieve } \\
\text { management }\end{array}$ \\
my studies & 2 & $\begin{array}{l}\text { Click on the multiple-choice question and the } \\
\text { corresponding option will appear } \\
\text { Click on the notebook title and in the } \\
\text { corresponding text box }\end{array}$ & $\begin{array}{l}\text { Achectations } \\
\text { expectieve } \\
\text { Achiectations }\end{array}$ \\
\hline
\end{tabular}

The client mobile phone first sets up a network connection (GPRS or Wifi), then calls the Google speech recognition API interface. After the Google speech recognition service recognizes, the response call back function is called to return. The string corresponding to the user's spoken language. In order to correctly judge the user's spoken language, this article uses LRC subtitles and colloquial recognition results to match. LRC is the abbreviation of English Lyric, which is a plain text format for recording lyrics in the form of labels. The 
system spoken language training module is in the LRC file. Contains the correct content of the user's spoken language, matches the recognized string with the correct string after the system's speech recognition, and feeds the matching result back to the user.

Spoken language matching, the key code is as follows:

@ Override

Protected void on Activity Result (int request Code, int result-Code, Intent data)

[//TODO Auto-generated method stub

// call back gets data from Google speech recognition

If $($ result Code $==$ RESULT_OK $)\{/ /$ Get the characters of the voice

Array List $<$ String > results $=$ data. Get String Array List Extra

(Recognizer Intent. EXTRA_RESULTS);

//User voice string

String user Result = " ";

For (int $i=0 ; i<$ results. Size ( ); $i++)\{$ User Result $+=$ results. Get $(i)\}$

// Get user voice corresponding LRC string

String lrc Result = get LrCStr ();

//Results match and red display

Match Str (user Result, lrc Result ;\}

\section{Resource library function implementation}

The resource library function server side uses ASP. The NET framework, the client and server communication Web Service protocol, using the Web Service protocol can reduce the complexity of the system and ensure the flexibility, robustness and maintainability of the system. Because the client uses the Android system, and there is no corresponding Web Service library on the Android system, the third-party software package ksoap2-android-assembly-2 needs to be introduced on the Android system. Web Service access is achieved through this package.

\section{Lock screen control function realization}

The lock screen control is the interface that opens when the system enters the screen saver state. The lock screen control interface is an ordinary activity display. The start lock screen control activity is implemented by the Service object and the Broadcast, and the Service background constantly listens to the screen dimming and brightening events. When the screen is darkened and brightened, the lock screen activity is started by Broadcast. The key codes are as follows: 
// Register lock screen to start broadcasting

Intent Filter Screen on Filter $=$ new Intent Filter (" android. intent.

Action. SCREEN_ON");

Lock Desk Service. This. Register Receiver

// start the service

If (intent. Get Action (. equals (" android. intent. action.

SCREEN_ON")) $\{$ M Key guard Manager = Key guard Manager $)$ context. Get System-

Service (Context. KEYGUARD_SERVICE);

M Key guard Lock $=m$ Key guard Manager. New Key guard Lock ("Zd Lock 1");

M Key guard Lock. Disable Key guard ();

//Open the default lock screen Activity

Start Activity ( lock intent) ;\}

\section{Conclusion}

The development of mobile technology provides a new way for English learning. This paper develops English listening and speaking self-learning software based on the current popular operating system Android. The software mainly realizes two functions of listening training and speaking training, including repetition in listening training. The spoken language matching function in function and spoken language training is the core function of the software. In order to facilitate the user to control the software, the utility functions such as lock screen control and group management are developed. The development of this software provides a new and convenient way for users to practice English listening and speaking.

\section{References}

Al-Otaibi, H. M., Alamer, R. A., \& Al-Khalifa, H. S. (2016). The next generation of language labs: can mobiles help? A case study. Computers in Human Behavior, 59, 342-349. http://dx.doi.org/10.1016/j.chb.2016.02.028

Bernstein, L. E., Eberhardt, S. P., \& Jr, E. T. A. (2014). Audiovisual spoken word training can promote or impede auditory-only perceptual learning: prelingually deafened adults with late-acquired cochlear implants versus normal hearing adults. Frontiers in Psychology, 5(5), 934. http://dx.doi.org/10.3389/fpsyg.2014.00934

Cavus, N., \& Ibrahim, D. (2017). Learning English using children's stories in mobile devices. British Journal of Educational Technology, 48(2), 625-641. http://dx.doi.org/10.1111/bjet.12427 
Filippo, C. L. D. (2016). When is auditory training not just learning how to listen? a holistic model of training functional auditory-based communication. Journal of Deaf Studies \& Deaf Education, 21(2), 41. http://dx.doi.org/10.1093/deafed/env041

James, P. S., Matthew, S., \& Samrachana, A. (2018). Constructing "Experts" Among Peers: Educational Infrastructure, Test Data, and Teachers' Interactions About Teaching. Educational Evaluation and Policy Analysis, 40(4), 586-612. http://dx.doi.org/10.3102/0162373718785764

Liu, T. Y., \& Chu, Y. L. (2010). Using ubiquitous games in an English listening and speaking course: impact on learning outcomes and motivation. Computers \& Education, 55(2), 630-643. http://dx.doi.org/10.1016/j.compedu.2010.02.023

Ou-Yang, F. C., Wu, W. V. (2017). Using mixed-modality vocabulary learning on mobile devices: design and evaluation. Journal of Educational Computing Research, 54(8), 1043-1069. http://dx.doi.org/10.1177/0735633116648170

Rice, T. (2010). Learning to listen: Auscultation and the transmission of auditory knowledge. Journal of the Royal Anthropological Institute, 16(s1), S41-S61. http://dx.doi.org/10.1111/j.1467-9655.2010.01609.x

Rockinson-Szapkiw, A. J., \& Walker, V. L. (2009). Web 2.0 technologies: facilitating interaction in an online human services counseling skills course. Journal of Technology in Human Services, 27(3), 175-193.

Wójcik, M. (2016). Potential use of augmented reality in lis education. Education \& Information Technologies, 21(6), 1-15. http://dx.doi.org/10.1007/s10639-015-9399-Z

Wu, H. K., Lee, W. Y., Chang, H. Y., \& Liang, J. C. (2013). Current status, opportunities and challenges of augmented reality in education. Computers \& Education, 62(2), 41-49. http://dx.doi.org/10.1016/j.compedu.2012.10.024

Yen, J. C., Tsai, C. H., \& Wu, M. (2013). Augmented reality in the higher education: Students' science concept learning and academic achievement in astronomy. Procedia - Social and Behavioral Sciences, 103, 165173. http://dx.doi.org/10.1016/j.sbspro.2013.10.322 